\title{
Estudo da miscibilidade de blendas de poli (ácido lático)/ poli (butileno adipato-co-tereftalato) preparadas pelo método de evaporação de solvente
}

\author{
Study of the miscibility of poly (lactic acid) / \\ poly (butylene adipate-co-terephthalate) blends \\ prepared by solvent-casting method
}

Fernanda Nardo Cobo ${ }^{1}$ Henrique de Santana ${ }^{1}$, Gizilene Maria de Carvalho ${ }^{1}$, Fábio Yamashita ${ }^{2}$

\footnotetext{
${ }^{1}$ Universidade Estadual de Londrina, Departamento de Química/CCE, Rod. Celso Garcia Cid, Pr 445 Km 380, Campus Universitário, CP 10.011, CEP: 86057-970, Londrina, PR, Brazil.

${ }^{2}$ Universidade Estadual de Londrina, Departamento de Ciências e Tecnologia de Alimentos/CCA, Rod. Celso Garcia Cid, Pr 445 Km 380, Campus Universitário - CP 10.011, CEP: 86057-970, Londrina, PR, Brazil.

e-mail: fe.nardocobo@gmail.com, hensan@uel.br, gizilene@uel.br, fabioy@uel.br
}

\begin{abstract}
RESUMO
A produção de blendas de PLA/ PBAT tem por objetivo superar as desvantagens que cada polímero possui individualmente, como fragilidade, limitações de processabilidade e custo. Neste estudo, investigamos a miscibilidade e as propriedades de filmes biodegradáveis de PLA - Poli (ácido lático), PBAT- Poli (butileno adipato-co-tereftalato) e de suas blendas com proporções de 95/5, 70/30, 30/70 e 5/95 de PLA/PBAT, respectivamente, produzidos pelo método de evaporação de solvente. As blendas produzidas foram caracterizadas pelas técnicas de espectroscopia Raman, calorimetria exploratória diferencial (DSC), termogravimetria (TGA), microscopia eletrônica de varredura (MEV), viscosimetria de soluções diluídas e ensaio mecânico de tração. A análise dos espectros Raman, temperatura de transição vítrea (Tg), e TGA indicaram imiscibilidade dos polímeros em todas as composições estudadas. As análises mecânicas apontaram para diminuição da rigidez das blendas com a adição do PBAT. Os valores dos parâmetros de interação obtido pela análise de viscosimetria de soluções diluídas, sugerem miscibilidade parcial da blenda contendo 5\% de PBAT. O estudo da miscibilidade e das propriedades das blendas de PLA/PBAT obtidas por evaporação de solvente possibilitarão a produção de novos materiais por diferentes técnicas visando novas aplicação para este material.
\end{abstract}

Palavras-chave: Poli (ácido lático). Poli (butileno adipato-co-tereftalato). Blendas. Misicibilidade. Viscosidade. Espectroscopia Raman.

\begin{abstract}
The production of PLA / PBAT blends aims to overcome the disadvantages that each polymer had individually, such as fragility, processability and cost limitations. In this study, we investigated the miscibility and properties of biodegradable films of PLA - Poli (lactic acid), PBAT-Poli (butylene adipate-co-terephthalate) and their blends with proportions of 95/5, 70/30, 30/70 and 5/95 PLA / PBAT, respectively, produced by the casting method. The blends produced were characterized by the techniques of Raman spectroscopy, differential scanning calorimetry (DSC), thermogravimetry (TGA), scanning electron microscopy (SEM), diluted solutions viscosimetry and mechanical tensile testing. The Raman spectra, glass transitions temperature (Tg), and TGA analysis indicated that the polymers are immiscible in the compositions studied. Mechanical analyzes point to a decrease in the stiffness of the blend with the addition of PBAT. The interaction parameters, obtained by viscosimetry analysis of diluted solutions, suggest partial miscibility of the blend with 5\% of PBAT. The study of the miscibility and properties of the PLA / PBAT blends obtained by solvent evaporation will enable the production of new materials by different techniques aiming at new applications for this
\end{abstract}


material.

Keywords: Poly (lactic acid). Poly (butylene adipate-co-terephthalate). Blends. Misicibility. Viscosity. Raman spectroscopy.

\section{INTRODUÇÃO}

Os plásticos sintéticos oriundos do petróleo tornaram-se materiais preciosos para a vida moderna, com ampla gama de aplicações. A produção anual de plásticos aumentou de 1,5 milhões de toneladas em 1950 para aproximadamente 359 milhões de toneladas em 2018 [1]. O sucesso dos plásticos é atribuído principalmente à grande versatilidade, durabilidade, resistência à corrosão e as suas propriedades intrínsecas. Contudo, o descarte inadequado desses materiais na natureza tem ocasionado sérios problemas ambientais, visto que eles se acumulam no ambiente por décadas [2,3].

Visando reduzir os impactos ambientais, grandes esforços têm sido realizados para produzir materiais poliméricos biodegradáveis que apresentem propriedades físico-químicas similares às dos plásticos convencionais. O poli (ácido lático) - PLA é um poliéster termoplástico, biodegradável e renovável que tem se destacado por sua boa processabilidade, versatilidade, biocompatibilidade e biodegradabilidade [4, 5]. O PLA é extensivamente utilizado na área biomédica, como na produção de implantes bioabsorvíveis, suturas cirúrgicas, scaffolds para engenharia de tecidos e em sistemas de liberação de fármacos [6]. Outra promissora aplicação do PLA é na produção de embalagens biodegradáveis [7]. Apesar de suas potencialidades, o PLA possui algumas desvantagens, como por exemplo, baixa flexibilidade e ductilidade e resistência ao impacto [8].

Um dos caminhos para melhorar as propriedades físicas do PLA e expandir suas aplicações é a produção de blendas poliméricas [9]. Blendas poliméricas são misturas físicas de dois ou mais polímeros com o propósito de produzir um material novo e com propriedades físicas distintas aos de origem [10]. Um dos polímeros mais promissores para a produção de blendas poliméricas com o PLA é o poli (butileno adipato-cotereftalato) - PBAT, principalmente por sua capacidade de melhorar a flexibilidade do PLA, sem prejudicar a biodegradabilidade do mesmo [11].

JIANG e colaboradores [12] produziram blendas de PLA/PBAT pela mistura mecânica dos polímeros no estado fundido. Através dos valores de temperatura de transição vítrea (Tg) obtidos pela análise mecânica dinâmica (DMA), a imiscibilidade das blendas PLA/PBAT foi constatada para todas as composições estudadas $(5,10,15$ e $20 \%$ de PBAT). Apesar da imiscibilidade das blendas PLA/PBAT, algumas propriedades mecânicas apresentaram melhoras, tais como, a tenacidade, o alongamento na ruptura e a resistência ao impacto [12]. YEH e colaboradores [13] estudaram a miscibilidade de blendas PLA/PBAT pelas técnicas de DSC e DMA. Por meio do deslocamento da Tg, os autores afirmaram que as blendas PLA/PBAT com composição igual ou menor que $2,5 \%$ de PBAT são termodinamicamente miscíveis [13]. Posteriormente, DIL, CARREAU e FAVIS [14] investigaram a morfologia e a miscibilidade de blendas PLA/PBAT utilizando técnicas microscópicas, DSC e análises reológicas. O estudo revelou uma miscibilidade parcial de PBAT em uma fase rica em PLA, no entanto, a elevação da massa molar do PBAT resultou em uma redução significativa da miscibilidade parcial [14]. WANG, RHIM e HONG [7] produziram blendas PLA/PBAT pelo método de casting. A miscibilidade e as propriedades térmicas, ópticas, mecânicas e de barreira ao vapor de água foram investigadas, os resultados de DSC e FT-IR indicaram a imiscibilidade das blendas PLA/PBAT, também foi observado que a adição de PBAT reduziu a fragilidade e melhorou a flexibilidade dos materiais [7].

Apesar das blendas PLA/PBAT serem extensivamente estudadas, poucos trabalhos na literatura analisam as propriedades destas blendas quando obtidas pelo método de espalhamento de solvente. A utilização deste método pode promover a obtenção de filmes com morfologias diversas, variando-se a concentração dos polímeros, taxa de evaporação do solvente, entre outras variáveis. Quando associada a outras técnicas experimentais, como por exemplo, a técnica de spin coating, eletrofiação e lixiviação de partículas, amplia as aplicações dos filmes obtidos [15-17].

Neste sentido, o presente trabalho tem por objetivo contribuir com o estudo da miscibilidade e das propriedades térmicas, mecânicas e morfológicas de blendas PLA/PBAT preparadas pelo método de evaporação de solvente, tendo como diferencial a utilização das técnicas viscosimetria de soluções diluídas e a espectroscopia Raman.

\section{MATERIAIS E MÉTODOS}

Poli (ácido lático), PLA Ingeo ${ }^{\mathrm{TM}}$ 4043D, com massa molecular média de 200 KDa adquirido da NatureWorks LLC (Blair, Nebraska, USA) e Poli (butileno adipato-co-tereftalo), PBAT, sob o nome comercial de Ecoflex ${ }^{\circledR}$ 
F BLEND C1200 [18], fornecido pela empresa BASF (Germany) [18] foram utilizados para produção das blendas. Clorofórmio (Biotec- Brasil) foi utilizado como solvente.

Os filmes de PLA, PBAT e das blendas PLA/PBAT foram produzidas pelo método de evaporação de solvente [7]. As blendas PLA/PBAT foram preparadas nas proporções 95/5, 70/30, 30/70 e 5/95 de PLA/PBAT a partir de soluções de $4 \%$ (massa/volume) dos polímeros dissolvidos em clorofórmio. As soluções foram agitadas por seis horas e posteriormente vertidas em placas de vidro e secas a temperatura ambiente por 24 horas. As amostras dos filmes produzidos foram denominadas PLA, PLA $955 \mathrm{PBAT}_{5}$, $\mathrm{PLA}_{70} / \mathrm{PBAT}_{30}, \mathrm{PLA}_{30} / \mathrm{PBAT}_{70}, \mathrm{PLA}_{5} / \mathrm{PBAT}_{95}$ e PBAT.

Os espectros Raman das amostras foram obtidos através do equipamento Microscópio Raman Confocal, marca Witec, modelo Alpha300 R, com laser de $532 \mathrm{~nm}$ e resolução espectral de $4 \mathrm{~cm}^{-1}$. A faixa de número de onda foi de 150 a $4000 \mathrm{~cm}^{-1}$. Imagens Raman foram obtidas com o auxílio de um microscópio confocal em diferentes espessuras da amostra e associadas com o espectro Raman.

Análises termogravimétricas (TGA) foram realizadas no equipamento Perkin Elmer TGA 4000, sob fluxo de nitrogênio com vazão de $20 \mathrm{~cm}^{3} / \mathrm{min}$. As amostras foram aquecidas no intervalo de temperatura de $30-800{ }^{\circ} \mathrm{C}$, com taxa de aquecimento de $20^{\circ} \mathrm{C} / \mathrm{min}$.

A calorimetria diferencial de varredura (DSC) foi realizada em um equipamento da Shimdzu, modelo DSC-60. As amostras dos filmes foram colocadas em panelas de alumínio e aquecidas de 30 a $200{ }^{\circ} \mathrm{C}$. A velocidade de aquecimento foi de $10{ }^{\circ} \mathrm{C} / \mathrm{min}$, sob uma atmosfera de nitrogênio com vazão de $50 \mathrm{~mL} / \mathrm{min}$. Para uma melhor visualização da temperatura de transição vítrea $(\mathrm{Tg})$ das amostras realizou-se uma segunda varredura no intervalo de 30 a $100^{\circ} \mathrm{C}$.

Os testes de resistência à tração foram realizados em uma máquina universal de ensaio (marca Emic, modelo DL2000 e célula de carga de 50 N), segundo as especificações da norma ASTM D-638 [19]. Foi analisada a tensão na força máxima, a deformação na ruptura e o módulo elástico.

A análise morfológica foi realizada por Microscopia Eletrônica de Varredura (MEV) utilizando um equipamento da marca Philips, modelo FEI Quanta 200 com voltagem de aceleração de $8.6 \mathrm{kV}$. As amostras foram fraturadas em nitrogênio líquido para a observação da superfície de fratura. As superfícies de fratura foram previamente cobertas com uma fina camada de ouro e a magnitude de observação foi de 2400 e 5000 vezes.

Um viscosímetro do tipo Cannon Fenske (marca Paragon Scientific Ltd-CFR 150) foi empregado para determinar a viscosidade relativa dos polímeros puros e das blendas PLA/PBAT. O intervalo de concentração investigado foi de $0,1 \mathrm{a} 2,0 \mathrm{~g} / \mathrm{dL}$, utilizando clorofórmio como solvente comum. Todos os experimentos foram conduzidos à temperatura constante de $23,0 \pm 0,5^{\circ} \mathrm{C}$.

A viscosimetria de soluções diluídas é uma técnica aplicada no estudo da miscibilidade de blendas poliméricas, visto que as interações mútuas entre os polímeros da solução afetam a viscosidade do sistema ternário (polímero $1+$ polímero $2+$ solvente). Se a blenda polimérica é imiscível haverá uma diminuição da viscosidade da solução devido às interações repulsivas entre as cadeias dos polímeros. Contudo, blendas poliméricas miscíveis apresentam aumento da viscosidade como consequência das interações atrativas entre as cadeias dos polímeros [20].

Para quantificar a miscibilidade de blendas poliméricas, CHEE [21] propôs uma expressão do parâmetro de interação, $\Delta \mathrm{B}$, quando os polímeros são misturados nas frações de peso $\mathrm{w}_{1} \mathrm{e} \mathrm{w}_{2}$, equação 1.

$$
\Delta \mathrm{B}=(\mathrm{b}-\mathrm{c}) /\left(2 \mathrm{w}_{1} \mathrm{w}_{2}\right)
$$

Onde: $c=w_{1} b_{11}+w_{2} b_{22}$, no qual $b_{11} e b_{22}$ são os parâmetros de interações individuais obtidos pela inclinação da reta das curvas de viscosidade reduzida versus concentração das soluções do polímero 1 e 2 . O coeficiente b está relacionado com o coeficiente de Huggins, Kh, equação 2.

$$
\mathrm{b}=\mathrm{Kh} \cdot[\mathrm{n}]^{2}
$$

Em sistemas ternários, o coeficiente b é dado pela equação 3 . O parâmetro de interação $b_{12 \text { é }}$ obtido pela inclinação da reta das curvas de viscosidade das soluções das blendas poliméricas.

$$
\mathrm{b}=\mathrm{w}_{12} \mathrm{~b}_{11}+\mathrm{w}_{22} \mathrm{~b}_{22}+2 \mathrm{w}_{1} \mathrm{w}_{2} \mathrm{~b}_{12}
$$

Usando os valores de $\Delta \mathrm{B}$, CHEE [21] definiu outro parâmetro de interação, $\mu$, dado pela equação 4 :

$$
\mu=\Delta \mathrm{B} /\left\{[\mathrm{n}]_{2}-[\mathrm{n}]_{1}\right\}^{2}
$$


Onde: $[\mathrm{n}]_{1}$ e $[\mathrm{n}]_{2}$ correspondem a viscosidade para soluções dos polímeros puros.

Se $\mu \geq 0$, a blenda polimérica é classificada como miscível. Porém, se $\mu<0$ a blenda polimérica é classificada como imiscível [22].

\section{RESULTADOS}

A estabilidade e a decomposição térmica dos filmes PLA, PLA 95 PBAT $5, \mathrm{PLA}_{70} / \mathrm{PBAT}_{30}, \mathrm{PLA}_{30} / \mathrm{PBAT}_{70}$, $\mathrm{PLA}_{5} / \mathrm{PBAT}_{95}$ e PBAT foram investigadas por TGA (Figura 1). Os dados correspondentes a T90 (temperatura observada em 10\% de perda de massa, Figura 1a) e Tmax (temperatura máxima de degradação, observados nas curvas de DTG, Figura 1b) estão registrados na Tabela 1. Pelos valores de T90, observa-se que a estabilidade térmica da amostra PLA é inferior a amostra PBAT. A diferença na estabilidade térmica dos materiais está relacionada provavelmente às diferenças estruturais das cadeias poliméricas [14].
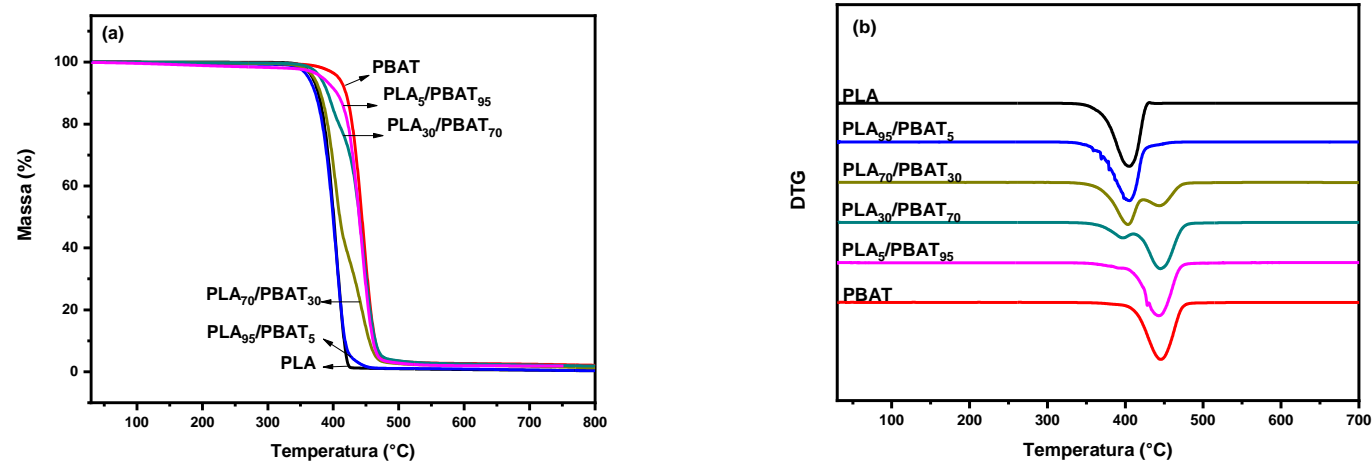

Figura 1: (a) Curvas de perda de massa em função da temperatura das amostras PLA, $\mathrm{PLA}_{95} / \mathrm{PBAT}_{5}, \mathrm{PLA}_{70} / \mathrm{PBAT}_{30}$, $\mathrm{PLA}_{30} / \mathrm{PBAT}_{70}, \mathrm{PLA}_{5} / \mathrm{PBAT}_{95}$ e PBAT, (b) Curvas da primeira derivada da perda de massa (DTG). Taxa de aquecimento de $20^{\circ} \mathrm{C} /$ min e vazão de $\mathrm{N}_{2}$ de $20 \mathrm{~mL} / \mathrm{min}$

O PLA e PBAT apresentaram um único evento de degradação, observados nas curvas de TG e DTG. O mesmo comportamento térmico foi observado na curva de DTG para as blendas PLA P $_{95} / \mathrm{PBAT}_{5}$ e $\mathrm{PLA}_{5} / \mathrm{PBAT}_{95}$, o que pode ser explicado pela pequena quantidade da fase dispersa, considerando que na curva de TG é possível visualizar a decomposição dos dois materiais em temperaturas diferentes. Para as blendas $\mathrm{PLA}_{70} / \mathrm{PBAT}_{30}$ e $\mathrm{PLA}_{30} / \mathrm{PBAT}_{70}$ observou-se, tanto na curva de TGA como na curva de DTG, dois eventos de degradação: o primeiro em aproximadamente $400{ }^{\circ} \mathrm{C}$ corresponde à degradação térmica do PLA e o segundo em torno de $443^{\circ} \mathrm{C}$ é referente à degradação térmica do PBAT. O fato dos valores de Tmax não apresentarem deslocamentos expressivos com relação aos valores de Tmax observados para os polímeros puros sugere a imiscibilidade das blendas com composição [14].

Tabela 1: Valores de T90 e Tmax das amostras PLA, PLA $95 / \mathrm{PBAT}_{5}, \mathrm{PLA}_{70} / \mathrm{PBAT}_{30}, \mathrm{PLA}_{30} / \mathrm{PBAT}_{70}, \mathrm{PLA}_{5} / \mathrm{PBAT}_{95}$, PBAT.

\begin{tabular}{c|c|c}
\hline Composição dos filmes & T90 $\left({ }^{\circ} \mathbf{C}\right)$ & Tmax $\left({ }^{\circ} \mathbf{C}\right)$ \\
\hline PLA & 377 & 404 \\
\hline PLA $_{95} / \mathrm{PBAT}_{5}$ & 373 & 405 \\
\hline PLA $_{70} / \mathrm{PBAT}_{30}$ & 380 & $403 / 444$ \\
\hline PLA $_{30} / \mathrm{PBAT}_{70}$ & 391 & $397 / 445$ \\
\hline PLA $_{5} / \mathrm{PBAT}_{95}$ & 406 & 443 \\
\hline PBAT & 419 & 446 \\
\hline
\end{tabular}

T90- Temperatura na qual há 10\% de perda de massa do material; Tmax-Temperatura máxima de degradação. 
$\mathrm{O}$ comportamento térmico dos filmes PLA, PLA $95 / \mathrm{PBAT}_{5}, \mathrm{PLA}_{70} / \mathrm{PBAT}_{30}, \mathrm{PLA}_{30} / \mathrm{PBAT}_{70}$, $\mathrm{PLA}_{5} / \mathrm{PBAT}_{95}$ e PBAT também foi investigado pela técnica de DSC. As Figuras 2(a) e 2(b) apresentam as curvas de DSC do primeiro $\left(30-200{ }^{\circ} \mathrm{C}\right.$ - taxa de $\left.10^{\circ} \mathrm{C} / \mathrm{min}\right)$ e do segundo aquecimento $\left(30-100{ }^{\circ} \mathrm{C}-\right.$ taxa de $\left.10^{\circ} \mathrm{C} / \mathrm{min}\right)$, respectivamente.
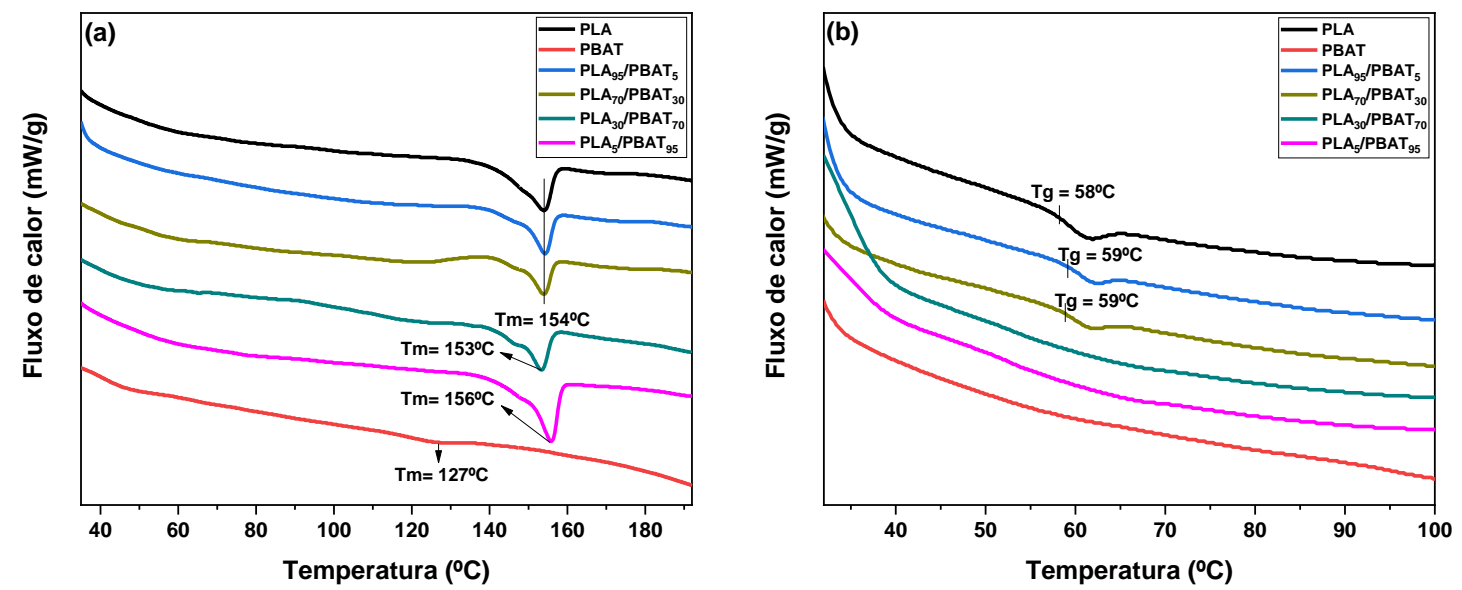

Figura 2: (a) Curvas de DSC do primeiro aquecimento $\left(30-200^{\circ} \mathrm{C}\right.$ - taxa de $10^{\circ} \mathrm{C} / \mathrm{min}$ e vazão de $\mathrm{N}_{2}$ de $\left.50 \mathrm{~mL} / \mathrm{min}\right)$ para as amostras de PLA, $\mathrm{PLA}_{95} / \mathrm{PBAT}_{5}, \mathrm{PLA}_{70} / \mathrm{PBAT}_{30}, \mathrm{PLA}_{30} / \mathrm{PBAT}_{70}, \mathrm{PLA}_{5} / \mathrm{PBAT}_{95}$ e PBAT. (b) Curvas de DSC do segundo aquecimento $\left(30-100^{\circ} \mathrm{C}\right.$ - taxa de $10^{\circ} \mathrm{C} / \mathrm{min}$ e vazão de $\mathrm{N}_{2}$ de $50 \mathrm{~mL} / \mathrm{min}$ ) para os filmes de PLA, $\mathrm{PLA}_{95} / \mathrm{PBAT}_{5}, \mathrm{PLA}_{70} / \mathrm{PBAT}_{30}, \mathrm{PLA}_{30} / \mathrm{PBAT}_{70}, \mathrm{PLA}_{5} / \mathrm{PBAT}_{95}$ e PBAT.

A Figura 2(a) revela que a amostra PLA apresenta um pico endotérmico bem definido em aproximadamente $154^{\circ} \mathrm{C}$ correspondente à temperatura de fusão cristalina $(\mathrm{Tm})$ e a amostra PBAT apresenta um pico muito discreto de fusão cristalina em torno de $127{ }^{\circ} \mathrm{C}$. De acordo com a literatura, o PBAT apresenta uma estrutura química aleatória composta por unidades rígidas de butileno tereftalato e unidades flexíveis de butileno adipato, a substituição de unidades aromáticas rígidas por unidades alifáticas flexíveis ocasiona um distúrbio cristalino, resultando em uma cristalinidade relativamente baixa [23, 24]. A Figura 2(a) também mostra que independente da proporção de PBAT nas blendas poliméricas, todas apresentaram um comportamento térmico semelhante ao PLA. Sendo assim, o pico endotérmico de fusão cristalina das blendas PLA/ PBAT está associado à cristalização de PLA e a adição de PBAT não teve efeito sobre a cristalinidade do PLA.

Com o objetivo de remover a história térmica anterior e tornar a temperatura de transição vítrea $(\mathrm{Tg})$ evidente, uma segunda curva de aquecimento de 30 a $100^{\circ} \mathrm{C}$ foi realizada para cada amostra. A Tg é um dos parâmetros mais eficientes no estudo da miscibilidade de blendas poliméricas. Pela análise da Tg dos materiais poliméricos, as blendas podem ser classificadas em miscíveis, imiscíveis ou parcialmente miscíveis. Blendas miscíveis apresentam uma única Tg, intermediário aos valores dos polímeros puros. Em contrapartida, as blendas imiscíveis apresentam dois valores de Tg, nas mesmas temperaturas que os polímeros puros. Por fim, as blendas parcialmente miscíveis apresentam mais de uma transição vítrea, em temperaturas intermediárias às Tgs dos polímeros puros [25].

A Figura 2(b) apresenta as curvas de DSC para o segundo aquecimento. Nesta análise não foi possível observar a Tg do PBAT, pois a temperatura de aquecimento foi acima da Tg do material que é aproximadamente $-30^{\circ} \mathrm{C}$ [7]. Por outro lado, é possível observar a Tg do PLA em aproximadamente $58{ }^{\circ} \mathrm{C}$. Analisando as curvas de aquecimento das blendas PLA/PBAT para as diferentes composições estudadas verifica-se que os valores da Tg das amostras $\mathrm{PLA}_{95} / \mathrm{PBAT}_{5}$ e $\mathrm{PLA}_{70} / \mathrm{PBAT}_{30}$ se apresentaram próximos dos valores da $\mathrm{Tg}$ do PLA, o que sugere que as blendas produzidas são termodinamicamente imiscíveis. Para as blendas $\mathrm{PLA}_{5} / \mathrm{PBAT}_{95}$ e $\mathrm{PLA}_{30} / \mathrm{PBAT}_{70}$ não foi possível observar a Tg, comportamento semelhante ao observado na literatura [26, 13].

As imagens de microscopia eletrônica de varredura (MEV) da superfície de fratura em nitrogênio líquido dos filmes PLA, PBAT e das quatro composições de blendas investigadas estão apresentadas na Figura 3. 

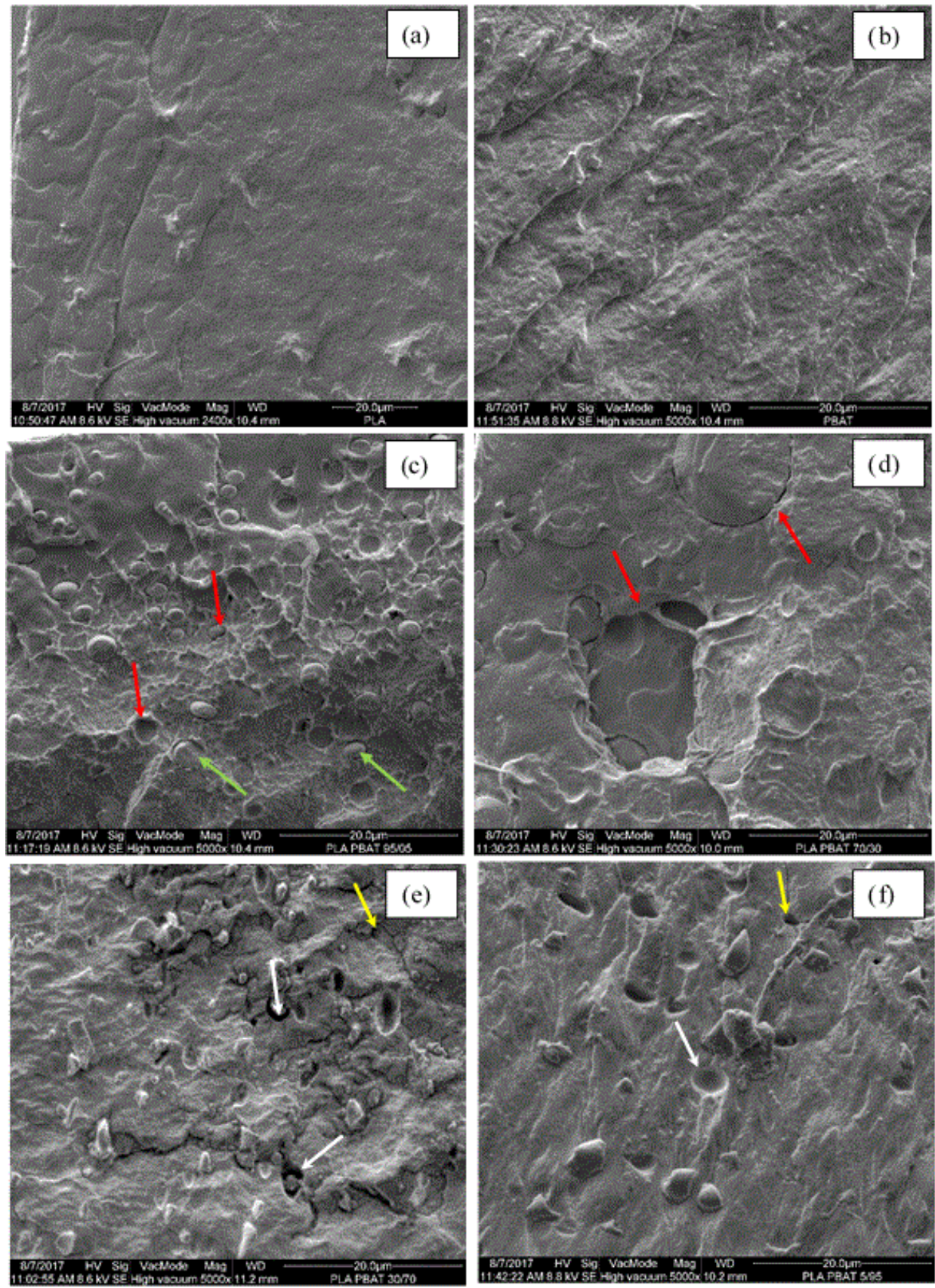

Figura 3: Imagens de Microscopia Eletrônica de Varredura da superfície da criofratura das amostras dos filmes (a) PLAaumento de 2400x, (b) PBAT (c), $\mathrm{PLA}_{95} / \mathrm{PBAT}_{5}$ (d), PLA 70 PBAT 30 (e), $\mathrm{PLA}_{30} / \mathrm{PBAT}_{70}$ (f) e PLA $/ \mathrm{PBAT}_{95}$ - aumento de $5000 \mathrm{x}$.

As imagens de MEV dos filmes PLA e PBAT podem ser observadas nas Figuras 3 (a) e (b), respectivamente. A imagens da superfície das fraturas das amostras mostram a relação entre a morfologia e o comportamento mecânico dos materiais. Para o PLA observa-se uma superfície de fratura lisa, característica de fratura frágil. Para o PBAT a superfície de fratura se apresenta rugosa e com aspecto mais arredondado, indicando que, apesar da fratura ser frágil (ocorrer abaixo da Tg do material), a elasticidade do material é responsável por maior deformação [26]. Estas características se refletem nos maiores valores de Módulo de Young, tensão na força máxima e menor valor de deformação na ruptura do PLA comparados aos valores apresentados pelo PBAT (Tabela 2).

Nas imagens (c), (d), (e) e (f) da Figura 3 pode-se observar que as blendas PLA $_{95} / \mathrm{PBAT}_{5}$, $\mathrm{PLA}_{70} / \mathrm{PBAT}_{30}, \mathrm{PLA}_{30} / \mathrm{PBAT}_{70}$ e PLA $/ \mathrm{PBAT}_{95}$ exibem morfologia típica de sistemas imiscíveis, apresentando separações de fases nítidas. Para as blendas $\mathrm{PLA}_{95} / \mathrm{PBAT}_{5}$ e $\mathrm{PLA}_{70} / \mathrm{PBAT}_{30}$ observa-se o PBAT disperso 
na fase de PLA. Na imagem da Figura 3 (c), PLA $_{95} / \mathrm{PBAT}_{5}$, é possível observar cavidades ovais nas regiões onde o PBAT apresenta descolamento da matriz (pull out) (setas vermelhas), com ausência de adesão interfacial e regiões onde o PBAT permaneceu aderido na matriz de PLA (setas verdes). O tamanho médio das partículas de PBAT disperso no PLA na blenda $\mathrm{PLA}_{5} / \mathrm{PBAT}_{95}$ foi de aproximadamente $2,4 \pm 0,5$ micrometros. A dispersão e a adesão de parte do PBAT na matriz de PLA contribui para a transferência de tensão entre as fases, resultando em um material que apresentou valores de módulo de Young e de tensão na força máxima superiores aos valores do PLA (Tabela 2). Para blenda PLA $_{70} / \mathrm{PBAT}_{30}$ (Figura 3d) o tamanho médio das partículas de PBAT dispersas na matriz de PLA foi de aproximadamente 5,8 \pm 1,6 micrometros, indicando que o PBAT se encontra mais aglomerado que na blenda $\mathrm{PLA}_{95} / \mathrm{PBAT}_{5}$. A aglomeração do PBAT contribui para a redução da área interfacial e se reflete na redução do Módulo de Young e da tensão na força máxima [27, 28].

As blendas $\mathrm{PLA}_{30} / \mathrm{PBAT}_{70}$ e PLA 5 /PBAT 95 apresentam inversão das fases, sendo que o PLA se encontra disperso na matriz de PBAT (Figuras 3e e 3f). A dispersão do PLA ocorre de maneira não homogênea e nota-se que a adesão interfacial entre os dois polímeros não é boa, observando-se cavidades ovais e vazios nas interfaces devido ao descolamento do PLA da matriz de PBAT (setas brancas). Também é possível observar que as superfícies da fratura destas amostras se apresentam mais irregulares e com trincas (setas amarelas). Não se observam regiões de interação entre as fases, diferente do verificado para amostras onde o PBAT é a fase dispersa (Figuras 3c e 3d). A falta de adesão entre as fases nestas amostras é responsável pela maior redução do Módulo de Young e da tensão na força máxima com o aumento da fração de PBAT.

Tabela 2: Propriedades mecânicas obtidas no ensaio mecânico de tração para os filmes de PLA, PBAT e suas blendas.

\begin{tabular}{l|c|c|c}
\hline Filme & $\begin{array}{c}\text { Tensão Força } \\
\text { Máxima (MPa) }\end{array}$ & $\begin{array}{c}\text { Deformação } \\
\text { na Ruptura (\%) }\end{array}$ & $\begin{array}{c}\text { Módulo } \\
\text { Young (MPa) }\end{array}$ \\
\hline PLA & $29,98 \pm 6,53$ & $5,61 \pm 1,78$ & $657,04 \pm 65,12$ \\
\hline PLA $_{95} / \mathrm{PBAT}_{5}$ & $25,08 \pm 7,52$ & $4,97 \pm 2,57$ & $765,67 \pm 85,16$ \\
\hline $\mathrm{PLA}_{70} / \mathrm{PBAT}_{30}$ & $16,77 \pm 2,87$ & $3,28 \pm 0,46$ & $542,76 \pm 34,28$ \\
\hline $\mathrm{PLA}_{30} / \mathrm{PBAT}_{70}$ & $7,35 \pm 0,93$ & $6,97 \pm 1,56$ & $331,82 \pm 33,41$ \\
\hline $\mathrm{PLA}_{5} / \mathrm{PBAT}_{95}$ & $6,82 \pm 1,14$ & $79,54 \pm 51,91$ & $132,58 \pm 13,58$ \\
\hline PBAT & $6,52 \pm 1,14$ & $58,54 \pm 29,47$ & $121,30 \pm 10,42$ \\
\hline
\end{tabular}

PLA- Poli (ácido lático), PBAT- poli (butileno adipato-co-tereftalo).

O PLA é um material frágil a temperatura ambiente, com baixa elongação na ruptura e alto módulo elástico. O filme de PLA puro apresentou tensão máxima de 29,98 ( $\pm 6,53)$ Mpa, deformação na ruptura de $5,61 \pm 1,78 \%$ e módulo elástico de 657,04 $\pm 65,12 \mathrm{MPa}$. Ao adicionar o PBAT ao PLA observa-se uma tendência de redução da tensão na força máxima e no módulo elástico e um aumento da \% de deformação na ruptura, indicando que o material se tornou menos frágil e mais flexível [11]. O aumento na elongação com o aumento da fração de PBAT é reflexo do aumento de ductilidade das blendas devido as propriedades elásticas do PBAT [26, 29].

A miscibilidade das blendas $\mathrm{PLA}_{95} / \mathrm{PBAT}_{5}, \mathrm{PLA}_{70} / \mathrm{PBAT}_{30}, \mathrm{PLA}_{30} / \mathrm{PBAT}_{70}$ e $\mathrm{PLA}_{5} / \mathrm{PBAT}_{95}$ foi avaliada por viscosimetria de soluções diluídas. Os valores de $[\mathrm{n}]$ e de $\mathrm{b}\left(\mathrm{b}_{11}, \mathrm{~b}_{22}\right.$ e $\left.\mathrm{b}_{12}\right)$ que correspondem a valores de interações encontram-se registrados na Tabela 3. Pelas equações 1 e 4 determinou-se o parâmetro $\Delta \mathrm{B}$ e $\mu$, respectivamente, para os sistemas compostos pela mistura de PLA/PBAT nas proporções 95/5, 70/30, $30 / 70$ e $5 / 95$. 
Tabela 3: Valores de viscosidade intrínseca [n], coeficientes de interação b11, b12, b22, $\Delta \mathrm{B}$ e $\mu$.

\begin{tabular}{l|c|c|c|c|c|c}
\hline Composição do filme & {$[\mathbf{n}]$} & $\mathbf{b}_{\mathbf{1 1}}$ & $\mathbf{b}_{\mathbf{1 2}}$ & $\mathbf{b}_{\mathbf{2 2}}$ & $\boldsymbol{\Delta B}$ & $\boldsymbol{\mu}$ \\
\hline PLA & 0,72454 & 0,43656 & - & - & - & - \\
\hline PLA $_{95} / \mathrm{PBAT}_{5}$ & 0,66372 & - & 0,42068 & - & 0,1394 & 1,4450 \\
\hline $\mathrm{PLA}_{70} / \mathrm{PBAT}_{30}$ & 0,54704 & - & 0,31861 & - & $-0,1365$ & $-0,6367$ \\
\hline $\mathrm{PLA}_{30} / \mathrm{PBAT}_{70}$ & 0,43745 & - & 0,13365 & - & $-0,0620$ & $-0,2893$ \\
\hline $\mathrm{PLA}_{5} / \mathrm{PBAT}_{95}$ & 0,35087 & - & 0,10985 & - & $-0,1715$ & $-0,8002$ \\
\hline PBAT & 0,26160 & - & - & 0,12596 & - & - \\
\hline
\end{tabular}

Como observado na Tabela 3 as blendas $\mathrm{PLA}_{70} / \mathrm{PBAT}_{30}, \mathrm{PLA}_{30} / \mathrm{PBAT}_{70}$ e $\mathrm{PLA}_{5} / \mathrm{PBAT}_{95}$ apresentaram valores de $\mu<0$, indicando a imiscibilidade das blendas para estas três composições. No entanto, a blenda $\mathrm{PLA}_{95} / \mathrm{PBAT}_{5}$ apresentou $\mu>0$, sugerindo interação entre os componentes da mistura.

A diferença de viscosidade dos dois polímeros pode contribuir para a ausência de interação entre as fases, como visto nas imagens de MEV. Entretanto a blenda PLA ${ }_{55} / \mathrm{PBAT}_{5}$ apresentou os coeficientes $\Delta \mathrm{B}$ e $\mu$ positivos, indicando que nesta composição podem estar ocorrendo interações entre os grupos carbonilas do PLA e do PBAT, favorecendo a miscibilidade parcial dos polímeros quando o PBAT se encontra em pequenas quantidades [30]. Esta observação é um indício de que a técnica de viscosidade pode ser útil quando outras técnicas não são adequadas para avaliar a miscibilidade de sistemas poliméricos.

A espectroscopia Raman foi utilizada para verificar as características químicas dos materiais e as possíveis interações entre o PLA e o PBAT nas blendas poliméricas. Os espectros Raman dos filmes PLA, $\mathrm{PLA}_{95} / \mathrm{PBAT}_{5}, \mathrm{PLA}_{70} / \mathrm{PBAT}_{30}, \mathrm{PLA}_{30} / \mathrm{PBAT}_{70}, \mathrm{PLA}_{5} / \mathrm{PBAT}_{95}$ e PBAT estão representados na Figura 4.

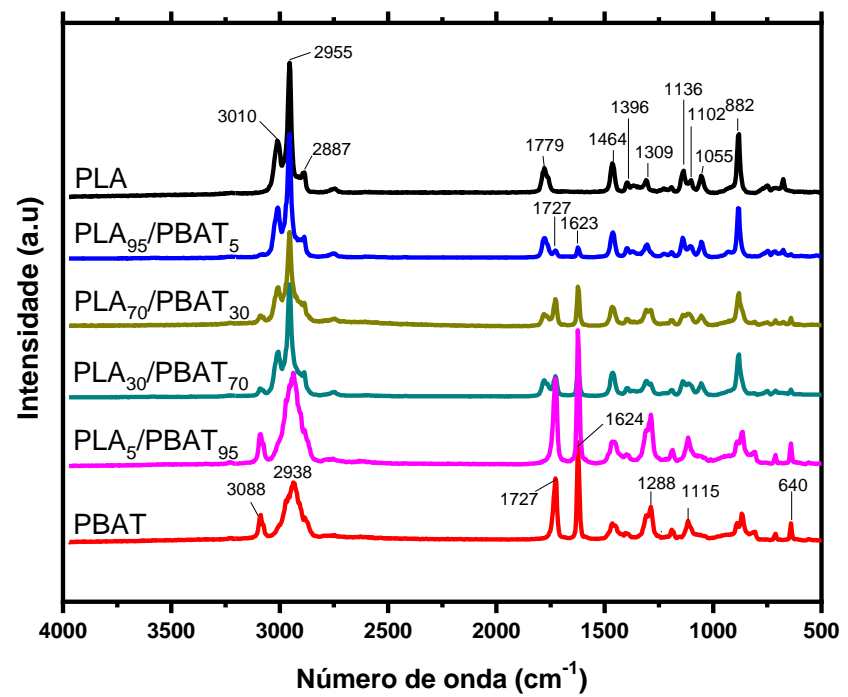

Figura 4: Espectros Raman para as amostras dos filmes PLA, PLA $95 / \mathrm{PBAT}_{5}, \mathrm{PLA}_{70} / \mathrm{PBAT}_{30}, \mathrm{PLA}_{30} / \mathrm{PBAT}_{70}$, $\mathrm{PLA}_{5} / \mathrm{PBAT}_{95}$ e PBAT.

Analisando o espectro Raman do filme PLA foi possível observar as principais bandas características do poli (ácido lático). As bandas associadas ao estiramento das ligações $\mathrm{C}-\mathrm{H}$ dos grupos $\mathrm{CH}_{3}$ apareceram entre 3100 e $2800 \mathrm{~cm}^{-1}$, em $1779 \mathrm{~cm}^{-1}$ observa-se a banda correspondente ao estiramento da carbonila $(\mathrm{C}=\mathrm{O})$ [31]. As deformações $\mathrm{C}-\mathrm{H}$ assimétricas e simétricas de grupos $\mathrm{CH}_{3}$ apareceram em 1464 e $1396 \mathrm{~cm}^{-1}$, respectivamente. Em $1309 \mathrm{~cm}^{-1}$ tem-se a deformação da ligação $\mathrm{C}-\mathrm{H}$, em $1136 \mathrm{~cm}^{-1}$ a deformação angular assimétrica no plano de grupos $\mathrm{CH}_{3}, 1102 \mathrm{~cm}^{-1}$ o estiramento da ligação C-O-C, $1055 \mathrm{~cm}^{-1}$ o estiramento da ligação C-C, por fim a intensa banda em $882 \mathrm{~cm}^{-1}$ corresponde ao estiramento C-COO [32].

Para o filme PBAT as principais bandas do espectro Raman podem ser observadas em: $3088 \mathrm{~cm}^{-1}$ correspondente ao estiramento da ligação C-H de anéis aromáticos, $2938 \mathrm{~cm}^{-1}$ estiramento da ligação C-H de grupos $\mathrm{CH}_{2}, 1727 \mathrm{~cm}^{-1}$ estiramento da carbonila $(\mathrm{C}=\mathrm{O})$, uma intensa banda em $1624 \mathrm{~cm}^{-1}$ atribuída a ligação $\mathrm{C}=\mathrm{C}$ de anéis aromáticos, $1288 \mathrm{~cm}^{-1}$ estiramento $\mathrm{C}-\mathrm{C}, 1115 \mathrm{~cm}^{-1}$ estiramento $\mathrm{C}-\mathrm{O}$ e $640 \mathrm{~cm}^{-1}$ vibração do 
anel aromático [33, 34].

Na Figura 4 observa-se que as blendas PLA/PBAT apresentaram a banda de vibração da carbonila de ambos os polímeros, sendo que, a intensidade dessas bandas variou de acordo com a proporção de cada polímero na blenda polimérica. Esse mesmo comportamento foi observado para as outras bandas de vibração que aparecem nos espectros Raman das blendas PLA/PBAT, tais como, a banda em 1624 e $640 \mathrm{~cm}^{-1}$, ambas características do PBAT.

Uma análise mais detalhada das interações entre os componentes da mistura foi realizada utilizando a obtenção de espectros em diferentes pontos e profundidades da amostra. A Figura 5 (a) apresenta os espectros Raman obtidos para a blenda $\mathrm{PLA}_{95} / \mathrm{PBAT}_{5}$. Cada espectro corresponde a uma região indicada pelas letras A-E nas imagens apresentadas na Figura 5 (b) e 5 (c), obtidas simultaneamente com os espectros Raman. Para as outras blendas os espectros e as imagens obtidas são semelhantes (não apresentadas aqui).
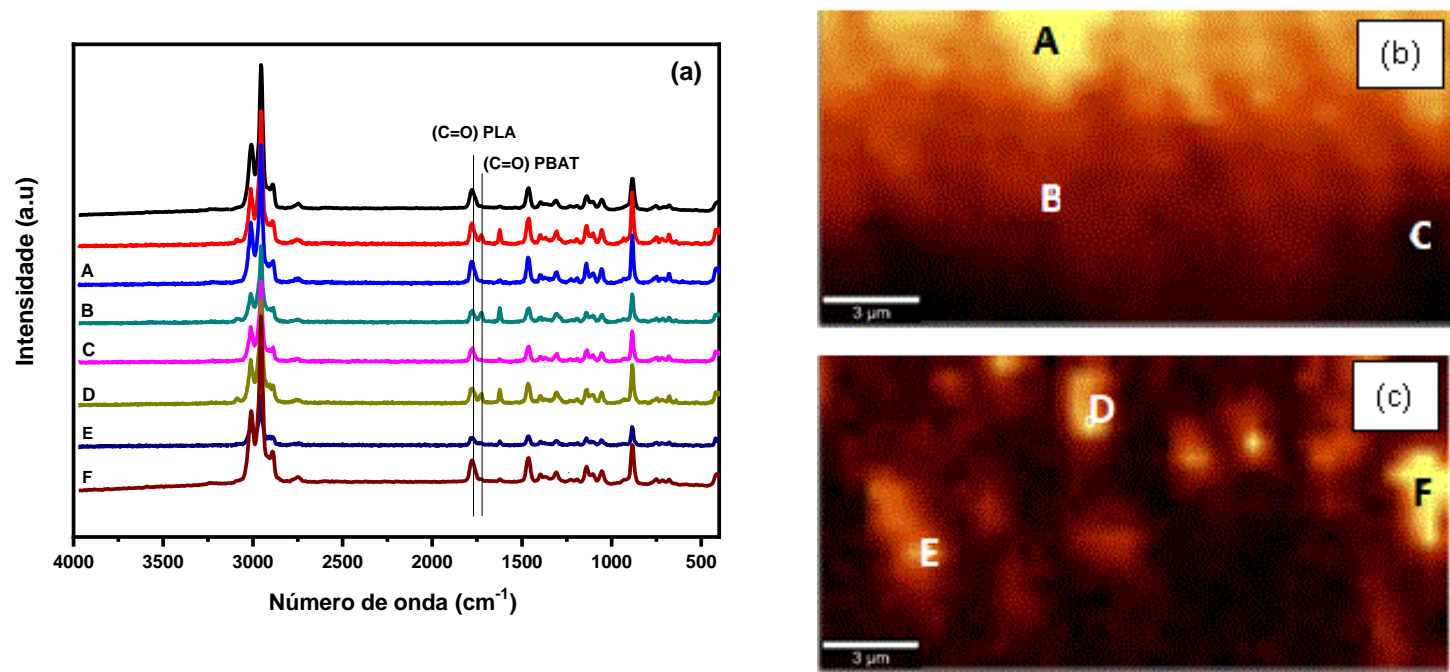

Figura 5: (a) Espectro Raman da blenda PLA $\mathrm{P}_{95} / \mathrm{PBAT}_{5}$ em diferentes profundidades da amostra (b) Imagem da região favorável ao PLA obtida por microscopia confocal e (c) Imagem da região favorável ao PBAT obtida por microscopia confocal. As letras indicadas nas imagens de microscopia confocal correspondem aos espectros de mesma letra na figura (a).

Os pontos A, B e C observados na Figura 5 (b) correspondem a regiões em que o PLA existe predominantemente (região favorável ao PLA). Para estas regiões observa-se no espectro Raman a presença da banda característica da carbonila do PLA $\left(1778 \mathrm{~cm}^{-1}\right)$ e a banda da carbonila do PBAT não é observada. Entretanto é possível observar a presença discreta da banda $\mathrm{em} 1622 \mathrm{~cm}^{-1}$ atribuída à ligação $\mathrm{C}=\mathrm{C}$ de anéis aromáticos presente no PBAT. Nos espectros referentes as regiões assinaladas como D, E e F, Figura 5 (c), é possível visualizar a banda $\mathrm{C}=\mathrm{O}$ do PLA e do PBAT, localizadas em $1779 \mathrm{~cm}^{-1}$ e $1727 \mathrm{~cm}^{-1}$, respectivamente. A razão da intensidade das bandas em 1779 e $1727 \mathrm{~cm}^{-1}$ é função da composição das blendas. Não foram detectadas alterações nas posições das bandas da carbonila para as misturas. Não é surpresa, uma vez que os valores do parâmetro de interação $\mu$ (Tabela 3), assim como os resultados de DSC, MEV e TGA encontrados para essas misturas indicaram imiscibilidade e/ou interações fracas entre o PLA e o PBAT [35-37]

\section{CONCLUSÕES}

Misturas de PLA e PBAT em diferentes composições foram analisadas com relação a miscibilidade utilizando as técnicas de DSC, TGA, viscosidade de soluções diluídas, MEV e espectroscopia Raman. As análises de TGA/DTG revelaram que as blendas possuem estabilidade térmica intermediarias aos valores observados para os polímeros puros. Entre as técnicas utilizadas apenas a viscosimetria de soluções diluídas foi capaz de detectar as interações na blenda PLA $_{95} / \mathrm{PBAT}_{5}$ que exibiram valores dos coeficientes de interação $\Delta \mathrm{B}$ e $\mu$ positivos. Estas interações foram confirmadas pelo elevado valor de Módulo de Young e de tensão na força máxima e pela melhor dispersão da fase PBAT na fase PLA observadas nas imagens de MEV, indicando miscibilidade parcial nesta composição. Estas observações demonstram a utilidade da viscosimetria de soluções diluídas para avaliar a miscibilidade de polímeros e auxiliar na investigação de misturas poliméricas obtidas a partir de soluções. Para as demais blendas PLA/PBAT analisadas $\left(\mathrm{PLA}_{70} / \mathrm{PBAT}_{30}, \mathrm{PLA}_{30} / \mathrm{PBAT}_{70}\right.$, 
$\mathrm{PLA}_{5} / \mathrm{PBAT}_{95}$ ) não foram detectadas interações moleculares que contribuíssem para a miscibilidade por nenhuma das técnicas utilizadas: Pela análise de DSC observou-se que não houve deslocamento da Tg do PLA nas blendas poliméricas PLA/PBAT. As curvas de TGA/DTG revelaram que as blendas possuem estabilidade térmica intermediarias aos valores observados para os polímeros puros. As imagens de MEV apresentaram morfologia típica de sistemas imiscíveis, com separações de fases entre a matriz e a fase dispersa. Os valores negativos de $\Delta \mathrm{b}$ e de $\mu$ obtidos pela análise de viscosimetria de soluções diluídas, os espectros Raman e as imagens de microscopia confocal confirmaram ausência de interações entre os polímeros. Consequentemente esta ausência de interação se reflete no comportamento mecânico destas blendas, que exibem menores valores de Módulo de Young e de tensão na força máxima.

\section{AGRADECIMENTOS}

Os autores agradecem ao Conselho Nacional de Desenvolvimento Científico e Tecnológico (CNPq-Brasil), Coordenação de Aperfeiçoamento de Pessoal de Nível Superior (CAPES - Brasil) pelo suporte financeiro, também ao Laboratório de Espectroscopia (ESPEC) e ao Laboratório de Microscopia Eletrônica e Microanálise (LMEM) da Central de Multiusuários da Universidade Estadual de Londrina.

\section{BIBLIOGRAFIA}

[1] PlasticsEurope, 2019. Plastics - the Facts 2019: An Analysis of European Plastics Production, Demand and Waste Data. 2019 (Retrieved from) https://www.plasticseurope.org/application/files/9715/7129/9584/ FINAL_web_version_Plastics_the_facts2019_14102019.

[2] HAMAD, K., KASEEM, M., KO, Y., et al., "Biodegradable polymer blends and composites: An Overview", Polymer Science, Ser. A, v. 56, pp. 812-829, 2014.

[3] HAIDER, T.P., VÖLKER, C., KRAMM, J., et al., "Plastics of the Future? The Impact of Biodegradable Polymers on the Environment and on Society", Angew. Chem. Int. Ed., v. 58, pp. 50-62, 2019.

[4] CASTRO-AGUIRRE, E., IÑIGUEZ-FRANCO, F., SAMSUDIN, H., et al., "Poly (lactic acid)- Mass production, processing, industrial applications, and end of life", Advanced Drug Delivery Reviews, v. 107, pp. 333-366, 2016.

[5] FARAH, S.; ANDERSON, D. G. LANGER, R. "Physical and mechanical properties of PLA, and their functions in widespread applications - A comprehensive review". Advanced Drug Delivery Reviews, v.107, pp. 367-392, 2016.

[6] NOFAR, M., SACLIGIL, D., CARREAU, P.J., et al., "Poly (lactic acid) blends: Processing, properties and applications". International journal of biological macromolecules, v. 125, p. 307-360, 2019.

[7] WANG, L.F., RHIM, J.W., HONG, S. "Preparation of poly(lactide)/poly(butylene adipate-coterephthalate) blend films using a solvent casting method and their food packaging application", Food Science and Technology, v. 68, pp. 454-461, 2016.

[8] RAQUEZ, J.M., HABIBI, Y., MURARIU, M., et al., "Polylactide (PLA)-based nanocomposites", Prog. Polym. Sci. n. 38, pp.1504-1542, 2013.

[9] RASAL, R.M., JANORKAR, A.V., HIRT, D.E. "Poly (lactic acid) modifications", Progress in Polymer Science, v. 35, pp. 338-356, 2010.

[10] PARAMESWARANPILLAI, J., THOMAS, S., GROHENS, Y. "Polymer Blends: State of the Art, New Challenges, and Opportunities”. In: Characterization of Polymer Blends, 1-5, 1. Ed. Weinheim: Wiley- VCH, 2015.

[11] AL-ITRY, R., LAMNAWAR, K., MAAZOUZ, A. "Rheological, morphological, and interfacial properties of compatibilized PLA/PBAT blends", Rheologica Acta, v. 53, pp. 501-507, 2014.

[12] JIANG, L., WOLCOTT, M.P., ZHANG, J. "Study of Biodegradable Polylactide/Poly (butylene adipateco-terephthalate) Blends", Biomacromolecules, v. 7, pp. 199-207, 2006.

[13] YEH, J.T., TSOU, C.H., HUANG, C.Y., et al., "Compatible and Crystallization Properties of Poly (lactic acid)/Poly(butylene adipate-co-terephthalate) Blends”, Journal of Applied Polymer Science, v. 116, pp. 680-687, 2010.

[14] DIL, E.J., CARREAU, P.J., FAVIS, B.D. "Morphology, miscibility and continuity development in poly(lactic acid)/poly(butylene adipate-co-terephthalate) blends”, Polymer, v. 68, pp. 202-212, 2015.

[15] KANG, Y., CHEN, P., SHI, X., et al., "Preparation of open-porous stereocomplex PLA/PBAT scaffolds and correlation between their morphology, mechanical behavior, and cell compatibility". RSC advances, v. 8, n. 23, p. 12933-12943, 2018.

[16] ROSENBERGER, A.G., DRAGUNSKI, D.C., MUNIZ, E.C. et al., "Electrospinning in the preparation 
of an electrochemical sensor based on carbon nanotubes" Journal of Molecular Liquids, v. 298, p. 112068, 2020.

[17] TIPDUANGTA, P., BELTON, P., FÁBIÁN, L., et al., "Electrospun Polymer Blend Nanofibers for Tunable Drug Delivery: The Role of Transformative Phase Separation on Controlling the Release Rate", Mol. Pharmaceutics, v.13, pp. 25-39, 2016.

[18] BASF, Product Information: Ecoflex® F-Blend C1200. Biodegradable polyester for compostable film, 2013.

[19] Standard Test Method for Tensile Properties of Plastics. ASTM International. D638.

[20] PARASHAR, P., RAMAKRISHNA, K., RAMAPRASAD, A.T. "A Study on Compatibility of Polymer Blends of Polystyrene/Poly(4-vinylpyridine)", Journal of Applied Polymer Science, v. 120, pp. 1729-1735, 2011.

[21] CHEE, K.K. "Determination of polymer-polymer miscibility by viscometry", Eur. Polym. J. v. 26, pp. 423-426, 1990.

[22] RAJULU, A.V., REDDY, R.L., RAO, G.B. et al., "Miscibility Study of Cellulose Acetate/Carboxylated Poly(vinyl chloride) Blend in Cyclohexanone by Viscosity, Ultrasonic Velocity, Refractive Index, and Polarizing Microscopic Methods", Journal of Applied Polymer Science, v. 81, pp. 557-561, 2001.

[23] WANG, H., WEI, D., ZHENG, A., et al., "Soil burial biodegradation of antimicrobial biodegradable PBAT films", Polymer Degradation and Stability, v.116, 14-22, 2015.

[24] CRANSTON, E., KAWADA, J., RAYMOND, S., et al., "Cocrystallization model for synthetic biodegradable poly(butylene adipate-co-butylene terephthalate)", Biomacromolecules, v. 4, pp. 995- 999, 2003.

[25] QUENTAL, A.C., CARVALHO, F.P., TADA, E.S. et al., "Blendas de PHB e seus copolímeros: miscibilidade e compatibilidade", Química Nova, v. 33, pp. 438-446, 2010.

[26] CHIU, H.T., HUANG, S.Y., CHEN, Y.F., et al., "Heat Treatment Effects on the Mechanical Properties and Morphologies of Poly (Lactic Acid)/Poly (Butylene Adipate-co-terephthalate) Blends", International Journal of Polymer Science, v. 2013, pp. 1-11, 2013.

[27] WANG, B., JIN, Y., KANG, K., et al., "Investigation on compatibility of PLA/PBAT blends modified by epoxy-terminated branched polymers through chemical micro-crosslinking", e-Polymers, v. 20, pp. 20-39, 2020.

[28] OGUZ, H., DIGAN, C., KARA, D., et al., "Development of PLA-PBAT and PLA-PBSA bio-blends: Effects of processing type and PLA crystallinity on morphology and mechanical properties", AIP Conference Proceedings, v. 2055, pp. 030003 1-5, 2019.

[29] GIGANTEA, V., CANESIB, I., CINELLIA, P., et al., "Rubber Toughening of Polylactic Acid (PLA) with Poly(butylene adipate-coterephthalate) (PBAT): Mechanical Properties, Fracture Mechanics and Analysis of Ductile-to-Brittle Behavior while Varying Temperature and Test Speed", European Polymer Journal, v. 115, pp. 125-137, 2019.

[30] PIVSA-ART, W., PAVASUPREE, S., NARONGCHAI, O., et al., "Preparation of Polymer Blends between Poly (L-lactic acid), Poly (butylene succinate-co-adipate) and Poly (butylene adipate-co-terephthalate) for Blow Film Industrial Application" Energy Procedia, 9, pp. 581-588, 2011.

[31] VANO-HERRERA, K., MISIUNB, A., VOGTA, C. "Preparation and characterization of poly (lacticacid)/poly(methyl methacrylate) blend tablets for application in quantitative analysis by micro Raman spectroscopy", J. Raman Spectrosc. v. 46, pp. 273-279, 2015.

[32] JARMELO, S., MARQUES, D.A.S., SIMÕES, P.N., et al., "Experimental (IR/Raman and 1H/13C NMR) and theoretical (DFT) studies of the preferential conformations adopted by 1 lactic acid oligomers and poly(L-lactic acid) homopolymer", J. Phys. Chem. B., v. 116, pp. 9-21, 2012.

[33] CAI, Y., LV, J., FENG, J. "Spectral Characterization of Four Kinds of Biodegradable Plastics: Poly (Lactic Acid), Poly (Butylenes Adipate-Co-Terephthalate), Poly (Hydroxybutyrate-Co-Hydroxyvalerate) and Poly (Butylenes Succinate) with FTIR and Raman Spectroscopy", J. Polym. Environ., v. 21, pp. 108-114, 2013.

[34] CAI, Y., LV, J., FENG, J., et al., "Discrimination of Poly(butylenes adipate-co-terephthalate) and Poly(ethylene terephthalate) with Fourier Transform Infrared Microscope and Raman Spectroscope", Spectroscopy Letters: An International Journal for Rapid Communication, v. 45, pp. 280-284, 2012.

[35] WARD, Y., MI, Y. "The study of miscibility and phase behavior of phenoxy blends using Raman spectroscopy", Polymer, v. 40, pp. 2465- 2468, 1999.

[36] REISE, M.O., SANTANA, H., BILCK, A.P., et al., "Characterization of coated biodegradable trays by spectroscopic techniques", Industrial Crops \& Products, v. 112, pp. 511-514, 2018. 
[37] DONG, J., OZAKI, Y. "FTIR and FT-Raman Studies of Partially Miscible Poly (methyl methacrylate)/Poly(4-vinylphenol) Blends in Solid States", Macromolecules, v. 30, pp. 286-292, 1997.

\section{ORCID}

Fernanda Nardo Cobo

https://orcid.org/0000-0001-7606-9133

Henrique de Santana

https://orcid.org/0000-0003-3145-9079

Gizilene Maria de Carvalho

https://orcid.org/0000-0001-9514-7897

Fábio Yamashita

https://orcid.org/0000-0002-9280-0683 